\title{
UNDERSTANDINGS OF EXPLORATORY SEARCH FOR EDUCATIONAL SEARCH ENGINE
}

\author{
Dr. Kamal EL GUEMMAT \\ ORCID: 0000-0002-1136-4841 \\ Faculty of Sciences Ben M'Sik, Hassan II University \\ Casablanca, Morocco \\ Dr. Sara OUAHABI \\ ORCID: 0000-0001-6478-7218 \\ Faculty of Sciences Ben M'Sik, Hassan II University \\ Casablanca, Morocco
}

Received: 28/03/2018 Accepted: 29/11/2018

\begin{abstract}
Exploratory learning depends on several e-learning technologies to enable students to direct their learning. However, the educational search engines of e-learning platforms are inspired by exploratory learning approach. Thus, their search is characterized by learning and investigation. The purpose of this paper is to explore what is considered to be deep connection between exploratory search and educational search engine since they are two complementary subjects. This relation remains at the heart of the development of exploratory learning and investigations. The paper concludes by highlighting the importance of the learning and investigation of knowledge in exploratory search for educational search engine and give other perspectives to improve this practice.
\end{abstract}

Keywords: Exploratory learning, e-learning, educational search engines, exploratory search, investigation.

\section{INTRODUCTION}

In traditional learning environments, the information available to the learner has been carefully selected, edited, or reworked to meet the needs of the learner. However, with the advent of communication networks, resources including those that are not intended for educational consumption, will be available to learners during exploratory learning (Ip et al., 2000; Ip \& Naidu, 2001) emphasized the need to rethink the problems of the availability of educational resources.

Exploratory learning is an exploratory pedagogical model. This last one is delivered through a variety of online learning technologies, including hypermedia, multimedia, search engines, digital audio and video, graphics, and standalone learning modules developed using a variety of creative tools (Dabbagh, N., 2007).

E-learning search engines uses exploratory learning approach and more particularly the exploratory search approach in the case where the exploratory nature of the human information retrieval behavior has been supported which allows students according to search and indexing mechanisms to direct their own learning through the process of discovery, or guided discovery thereby the student learns the facts, concepts, and procedures. Due to exploratory search, the focus is on information or resources and the challenge is the effective discovery of resources while avoiding inappropriate resources. 
According to the researches consulted, there is not an exhaustive work discovering the deep relations between these two subjects. Yet, what seems, they are very connected, and one completes the other, and their relation remains at the heart of the development of exploratory learning and investigations. From where comes the idea to work on it.

Thus, this paper will focus on educational search engines and their exploratory search. There are several features that can supported which will be treated in detail throughout this paper.

This paper aims to explore what is believed to be a deep connection between exploratory search and educational search engine. The paper defined in the second section exploratory pedagogy for educational search engines. In the section three the paper traits the deep relations between the characterizing features of exploratory search and educational search engine that remains central to education developments. Later in the fourth section the paper discusses findings and suggestions. The paper concludes by highlighting the importance of the learning and investigation of knowledge in exploratory search for educational search engine and give other perspectives to improve this practice.

\section{DEFINING EXPLORATORY SEARCH FOR EDUCATIONAL SEARCH ENGINE}

Education is the process of teaching and learning in preschool, elementary school, high school then college, university, or apprenticeship; where the knowledge, skills, values, beliefs, and habits of a group of people are transferred from one generation to another through storytelling, discussion, teaching, training, or research. Any experience that has a formative effect on the method by which someone thinks, feels, acts, considers themselves educational. Education is often conducted under the guidance of others, but the learner can also learn by himself (self-learning). However, science and art are interested in finding the best way to educate, it's called pedagogy.

Exploratory pedagogy is based on the theoretical construction of discovery or inquiry learning. In this type of pedagogy students receive scientific inquiries or genuine problem in a given content area and are invited to generate a hypothesis, gather relevant information using a variety of resources and provide solutions, action plans, recommendations, and interpretations of situations (Dabbagh \& Bannan-Ritland, 2005).

Exploratory uses of instructional technology allow students to direct their own learning. Through the process of discovery, or guided discovery, the student learns facts, concepts, and procedures (Department of Education USA, 1993).

Exploratory search has been defined as more complex information retrieval activities that may be required by users who do not have clearly defined goals, have complex changing needs, or use a poorly indexed system (White, R. W. et al, 2006a). White and Roth in their review of exploratory search maintained this perspective, broadening it slightly to support that learning "is not only about knowledge acquisition, but rather the development of higher-level intellectual capabilities within a particular subject area" (White, R., \& Roth, R.A., 2009).

Also, Vakkari maintains this connection to both learning objectives and investigative search, he suggests that inquiry search is invoked "when actors are exploring possible conceptualizations of their topic" and that learning is illustrated by increasing the completeness and specificity of the mental model of the subject of the researcher as the search progresses (Vakkari, P., 2010). Kim's research had the same goal "to foster learning or investigation" (Kim, J., 2009).

The authors (Wildemuth, B.M. et al, 1995) have defined it as follows: "in exploratory search, users generally combine querying and browsing strategies to foster learning and investigation". Another definition of 
(White, R., \& Roth, R.A., 2009) "Exploratory search is a type of information seeking and a type of sensemaking focused on the gathering and use of information to foster intellectual development".

Exploratory search is characterized by learning and investigation cf. figure 1 (Marchionini, G., 2006). The acquisition of knowledge, understanding, interpretation of ideas, analyzes, synthesis and evaluation are all components of ongoing learning processes supported by exploratory search.
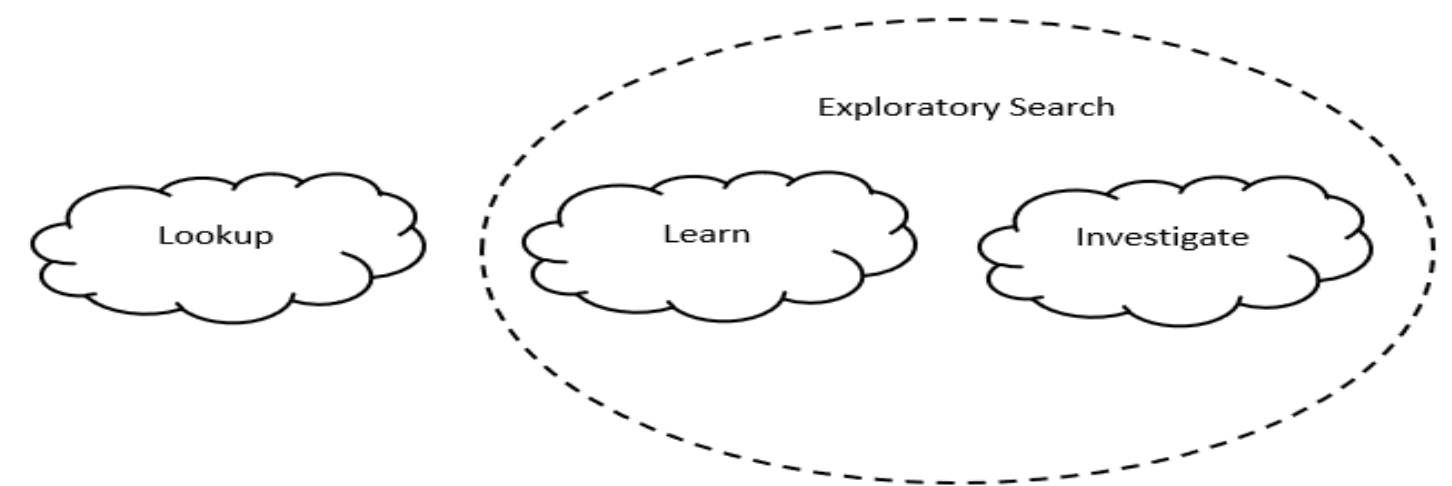

Fact retrieval
Known item search
Navigation
Transaction
Verification
Question answering

\begin{tabular}{l} 
Knowledge acquisition \\
Comprehension/Interpretation \\
Comparison \\
Aggregation/Integration \\
\hline
\end{tabular}

Accretion

Analysis

Exclusion/Negation

Synthesis

Evaluation

Discovery

Planning/Forcecasting

Transformation

Figure 1. Search activities

Marchionini suggests some of these possible activities, including: aggregation, comparison, and evaluation (Marchionini, G., 2006). Under these conditions, simple keyword search cannot support users effectively and convoluted user coping strategies have been recorded, implying an iterative tentative guessing of keywords (Pirolli, P., \& Card, S., 1995). Clearly, what users require is a search method that can involve more browsing and exploration activities to achieve their goals. This has been the basis for developing exploratory search interfaces (ESI) that support many rich search modes.

Exploratory pedagogy is delivered through a variety of online learning technologies, including hypermedia, multimedia, digital audio and video, graphics, and standalone learning modules developed using a variety of creative tools, search engines. From the point of view of educational search engines, its overriding objectives resonate with many similarities to the development of learner searching. Providing a link to a search engine in the course site allowing students to search for web resources to promote exploration.

However as quoted before, there is not an exhaustive work discovering the deep relations between these two subjects. Yet, what seems, they are very connected, and one completes the other. This relation remains at the heart of the development of exploratory learning and investigations. In the following of this paper. The focus is on several features of exploratory search and their deep relations with educational search engines. 
This section explores the conceptualization of exploratory search for educational search engine. The features of exploratory search will be represented within the concepts of cognitive and behavioral (Wildemuth, B. M., \& Freund, L., 2012).

In the first place will be treated the concepts of exploratory search before moving to the features of educational search engines and at the end of this section there are a comparative table that defines the different characteristics that represent the deep relations between exploratory search and educational search engines. Characteristics in the cognitive category include those that involve the reasoning associated with the development and conduct of exploratory search, while the characteristics of the behavioral category focus on behaviors that may be observed during exploratory search.

\section{Cognitive}

This section begins by showing the evidence of the main feature of exploratory search that is learning, and investigative search goals. It is clear that learning and inquiry are widely accepted objectives for exploratory search. Regarding the other characteristics of the exploratory search, that will be presented in the following will be considered secondary to the last.

There is that of "General" which designates conceptually broad search subject so the description of the tasks are vague and ill-defined. Several authors have described this characteristic as the abstract of search objectives so that search to be exploratory (Aula, A., \& Russell, D.M., 2009; Li, Y., 2009). For example (Glowacka, D. et al.,2013) have proposed a search engine supporting this principle, they have found an appropriate abstract level on which it is convenient for users to direct their search.

There is also the characteristic of uncertainty. Several studies have associated a certain level of uncertainty with exploratory search. The challenge is clear to build uncertainty in an exploratory search task. Uncertainty can be found as a characteristic of the information needs underlying the task (Alhenshiri, A. et al., 2012) or as the characteristics of the subject (Du, J.T., \& Evans, N., 2011) or as a characteristic of the sources that could solve the information needs (White, M.D., \& Iivonen, M., 2001) or as a characteristic of the search results (Niu, Y., \& Winter, S., 2006).

However, the multi-facet feature considers the description of the search task as multi-faceted which means "includes multiple aspects or a number of concepts" (Kintsch, W., 1998). Most often the description of the search task includes several subtasks (Diriye, A. et al., 2010). (Toms, E.G. et al., 2008) have analyzed this characteristic in detail; they found two different multi-face task structures. The first is a parallel structure, in which the different facets are "on the same level in a conceptual hierarchy". The second type of structure is the hierarchical one characterized by "a single concept for which multiple attributes or characteristics are sought".

Faceted navigation is an example of a class of exploratory search interfaces (ESI), that has metadata attributes as selectable categorized option sets. By modeling the field of information through a faceted structure, direct manipulation can be used to build queries. So, when a user is unclear about the appropriate terminologies or metadata are unpredictable, they do not have to estimate search terms, but can make a selection to build their queries. Through this additional support, faceted browsers can be considered as a type of ESI (Wilson, M. L., 2007). For example (Halvey, M. et al., 2014) shows the importance of using multi-faceted recommendations for video search tasks. 
The last feature that will be discussed in this section is that of cognitive behavior (Qu, Y., \& Furnas, G.W., 2006; Qu, Y., \& Furnas, G.W., 2008) identified a number of other information or cognitive behaviors frequently accompanying exploratory search behaviors. These include sensemaking, organizing and analyzing the results of search, making the decision. There are several researches increasing the discussion of teaching by the indication that comparison is a cognitive behavior, is a part of the exploratory search process. (Jansen et al., 2009) and (Wu et al., 2012) extend this idea even further, using a formal taxonomy of cognitive learning to develop search tasks. For example (Klouche et al., 2015) allow incremental exploration and sensemaking of large information spacing by the design of Exploration Wall, a touch-based search user interface. Shneiderman and his colleagues created a 'dynamic query' interface that uses mouse actions to immediately update the display to engage information seekers in the search process to help them perform multiple tasks in parallel and see the immediate impact of their decisions (Shneiderman, B. and Plaisant, C., 2005). In the case of (Kammerer, Y. et al., 2009) participants using the exploratory interface had a significantly higher cognitive load than the basic participants. One possible explanation for the higher cognitive load caused by the exploratory interface is the greater amount of cognitive processing during exploratory search because of the additional labels presented in addition and the relevant feedbacks.

\section{Behavioral}

This section focuses on behaviors that may be observed during exploratory search. There are three characteristics of the behavioral concept; Open-ended, Target multiple items / documents, dynamic and evolve over time. For the first, there is a difference between open or closed question. In the first studies of Marchionini (Marchionini, G., et al., 1993) include open-ended general information tasks and open-ended issues, described as a question "for which there was no specific answer". The characteristic of the open-ended search task often implies that the search target is multiple items / documents. (Marchionini, 2006) explicit in his observation that learning-oriented search returns a set of documents.

For the second, the characteristic of the open-ended search task often implies that the search target is multiple items / documents. (Marchionini, 2006) explicit in his observation that learning-oriented search returns a set of documents. (Ramdeen, S., \& Hemminger, B., 2012) asked his subjects to retrieve a particular number of items for each task.

For the third and in his recent research on the facets of search tasks, Li (Li, Y., 2009) identifies time (length) as a generic facet of an exploratory search task. It can be short or in long term. (Kammerer, Y. et al., 2009) found that there is a significant effect of the exploratory search interface on the time taken for search tasks.

\section{Educational Search Engine}

This section begins with the features of educational search engines before guessing the relationship with exploratory search. Educational search engines are a kind of extension and development of generic search engines to meet the specific needs of the disciplines of different users. Online learning systems interact with educational applications of search engines to overcome the limitations of general search. E-learning-based search engines offer many advantages to find, share, reuse and adapt learning object (El Guemmat, K., \& Ouahabi, S., 2018).

Like the generic search engines. The general work process is divided into several parts of which three processes are essential:

$>$ Indexing process: Indexing consists of summarizing a thing by characteristic elements from that thing to classify it and bring it closer to similar things.

$>$ Searching process: Comparison between the representation model of the request's index and the document.

$>$ Evaluation of the answer: Evaluate the response, to ensure that the approach taken by the system meets or not the need of the user, according to a degree of satisfaction. 
These indexing and searching techniques can be classical, semantic, by metadata or hybrid mixing two or more techniques. In the following there is a description of each technique:

$>$ Classic: This technique indexes only by keywords. It allows to find the most repeated words with their weight, follows the technique based on frequency of occurrences and technique based on Term Frequency $(\mathrm{TF}) *$ Inverted Document Frequency (IDF). The index can yield to some improvements like stop list to avoid the useless words, lemmatization to keep the source and to eliminate the endings. For the part of representation, it can influence on the meaning of the index retained, depending on the case, one of these models can be chosen. There is the vector model, the boolean model, the probabilistic model, the connectionist model, the bayesian inferential networks model, the latent semantic indexing model and there is the language model.

$>$ Semantics: This technique indexes by keyword and meaning at the same time to disambiguate the meanings of words (El Guemmat, K. et al., 2015). There are several indexing techniques, each of which has its advantages and disadvantages. There is the corpus technique, there is the linguistic structures, the ontologies technique, the thesaurus technique, the computerized dictionaries technique and there is the lexicons technique. For the representation and searching part there are also several techniques and each one can be used in a specific context. For example, there is the conceptual graphs model, the frame languages model, the semantic networks model, the topic maps model and there is the description logics model.

$>$ Metadata: The Metadata technique allows indexing only the course structure without going into the course content. It follows the different standards. For example, the LOM standard that confirmed its usefulness for learning object searching (Valiente et al, 2015).

> Hybrid: It combines several techniques (Biletskiy et al., 2012) to reap the benefits of each. it helps to arrive at more efficient educational search engines either at the level of indexing or searching.

In parallel with the indexing and searching stage, there is another important step in evaluating and improving searching. Like searching type, ranking results, query enrichment, profile, learning object language and implementation platform. These modernizations for educational search engines are pushing students to direct their studies through exploratory learning. The educational search engines have several characteristics that can correspond to exploratory search. These characteristics will be mentioned in the following.

Most learners use general or keywords-search engines to find learning materials related to their studies, but it seems less useful because of the time and effort that students must spend to find items related to their learning needs and preferences. General search cannot and probably should not meet the specific needs of disciplines (Hassan, S. \& Mihalcea, R., 2009) the need for amelioration is an important challenge in this case (Zhao, J. et al., 2008).

Semantic search engines overcome the problems that have been posed by classical search engines (Antoniou, G. van Harmelen. and F., 2004). Two major benefits of this transformation; the search is by meaning and the learning objects displayed in search results have a semantic relation with the need. We will mention three cases which have been able to affirm this statement (El Guemmat, K. et al., 2015; Lee, M. C. et al., 2008; Smine, B. et al., 2011). These search engines are well evaluated according to the precision, recall and $\mathrm{f}-$ measure measurements. The second benefit is the deduction of the appropriate semantic context for the search (Gluz, J. C. et al., 2016).

Additionally, and in order to tie more the search; the aspect of the description of the search task as multifaceted means that "includes multiple aspects or a number of concepts" (Kintsch, W., 1998). Clearly, what demanding users is a search method that may involve more browsing and exploration activities to achieve their goals. This has been the basis for developing exploratory search interfaces (ESI) that support many rich 
search modes (Wilson, M. L., 2007). Implications for interface design (content, browsing, navigation) are discussed in (Miwa, M., \& Kando, N., 2007).

Shneiderman and his colleagues created an interface "dynamic query" that uses mouse actions to immediately update the display to engage information seekers in the search process (Shneiderman, B. and Plaisant, C., 2005).

Also, the tag-based search help to find related resources we quote for example (Furini et al., 2017). And finally, the personalization of the search has been shown to be helpful in retrieval learning materials (Rahman et al., 2017). In the table below, there is a comparison that gathers and summarizes all the characteristics mentioned before. With a try to bring them near, the maximum possible, with adequate features in educational search engine side.

The characteristics of exploratory search are divided into two parts; cognitive and behavioral. In which there is many exploratory search tasks focus on learning and investigative search goals; they are general (rather than specific); they are multi-faceted and may be procedurally complex; they involve uncertainty and are motivated by ill-defined or ill-structured problems and they are often accompanied by other information or cognitive behaviors, such as sensemaking. They are open-ended; often target multiple items/documents and they are dynamic and evolve over time. For the characteristics of educational search engines are as follows: keywords, meaning, faceted browsing, navigation, interface design, dynamic query, tagging, personalized search.

Table 1. Exploratory search \& Educational search engine

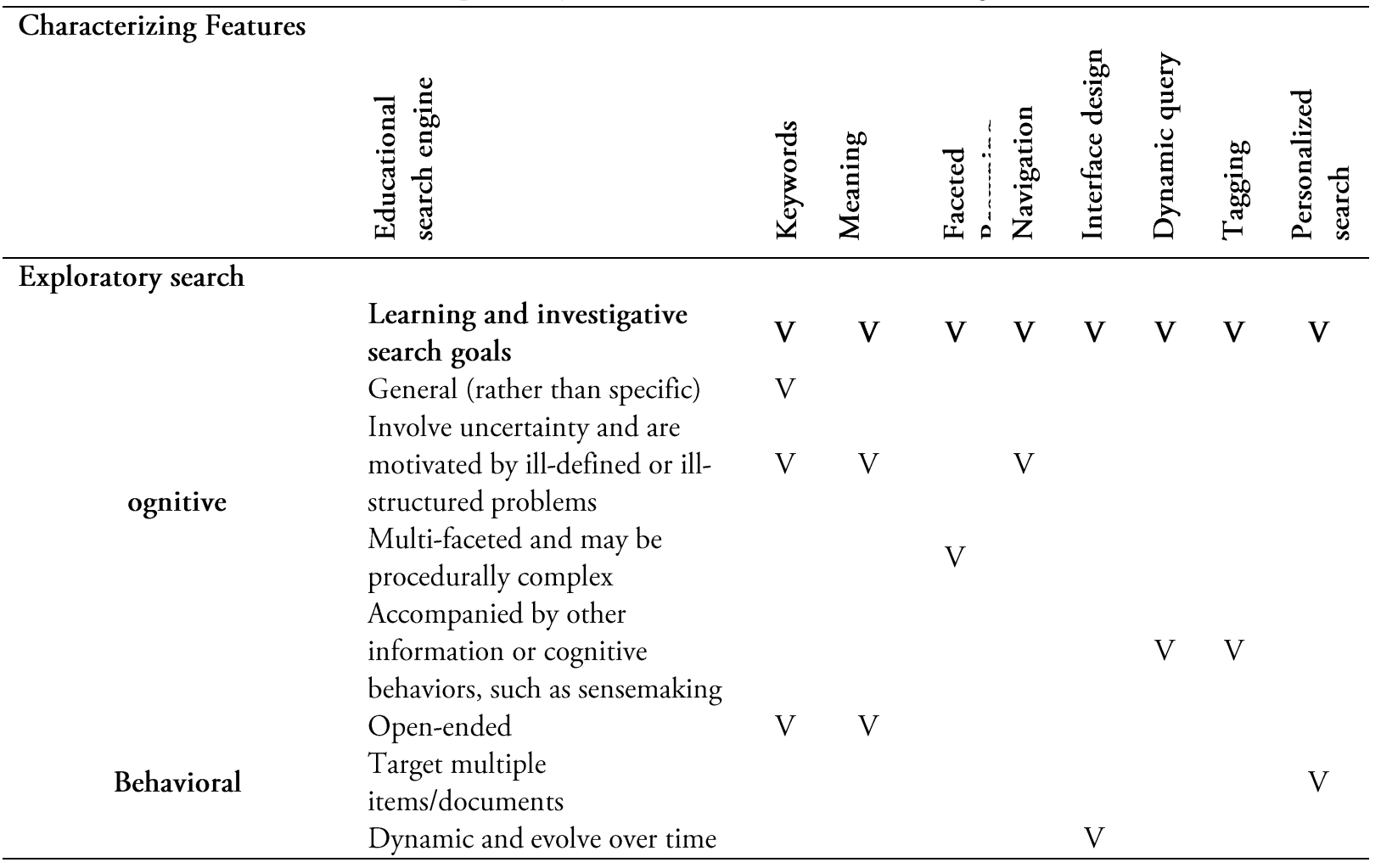




\section{FINDINGS AND SUGGESTIONS}

This paper finds that the first characteristic of exploratory search depends on all the characteristics of educational search engines. This is normal because it is the main exploratory feature involving the two main types of search activities: learning and investigation (Marchionini, G., 2006). For the other features he also finds that:

$>$ The general characteristic depends of search engine's keywords characteristics, since this feature abstract the objectives of the search (Aula, A., \& Russell, D.M., 2009; Li, Y., 2009).

$>$ The characteristic of uncertainty depends on the search engine keywords, meaning and navigation, since the coupling of these characteristics may associate a certain level of uncertainty with exploratory search (Alhenshiri, A. et al., 2012; Du, J.T., \& Evans, N., 2011; White, M.D., \& Iivonen, M., 2001; Niu, Y., \& Winter, S., 2006).

$>$ The multi-faceted feature is associated with faced browsing in search engine side, that can guarantee that the search task description includes multiple subtasks (Diriye, A. et al., 2010).

$>$ For the cognitive feature depends on dynamic query and tagging search engine side (Shneiderman, B. and Plaisant, C., 2005; Kammerer, Y. et al., 2009).

$>$ The Open-ended feature depends on keywords and mining in search engine side, since the openended questions, described as question "for which there was no specific answer" (Marchionini, G., et al., 1993).

$>$ The characteristic target multiple items/documents (Marchionini, 2006) depend on the personalized search in search engine side.

$>$ The characteristic "Dynamic and evolve over time" depends on the design of the search engine side interface (Kammerer, Y. et al., 2009).

This paper finds that exploratory search depends on educational search engines for learning and knowledge inquiry. From the perspectives of exploration, learning and investigation have an important place for many students looking for knowledge.

This result shows that the exploration principle is more inclined to rather investigation methods and that the student must make a cognitive effort to find the desired information. Yet, the behavior of students it's not the same, some of them are not ready all the time to make cognitive efforts; from where comes the need to think of other alternative solutions that can remedy this scourge.

It is accurate that we can customize the exploratory course (Wolfram, D., \& Dimitroff, A., 1998; Li, Y., 2009; Kammerer, Y. et al., 2009) and that exploratory research facilitate an increased level of interaction with information. But it is not clear that if researchers are forced to find a number of elements pushes naturalistic exploratory search behavior or results. Thus, more research is needed to investigate the potential effects of this practice (Wildemuth, B. M., \& Freund, L., 2012).

Given the characteristics of exploratory search tasks, (White, R. et al., 2006b) claim that measurement of "interaction behaviors, cognitive load, and learning" is suitable. While many of the studies reviewed in (Wildemuth, B. M., \& Freund, L., 2012) do assess interaction behaviors, fewer assess cognitive load or learning. However, the development of valid evaluation methods is critical to progress in the field (Wildemuth, B. M., 2007).

On the other hand, according to the comparison conducted by (El Guemmat, K., \& Ouahabi, S., 2018) most educational search engine do not adopt the principle of exploratory search especially the principle of behavior so interaction; otherwise the measures of relevance of these engines are based on the recognized measures in the field of standard search engines (precision, recall, f-measure). For example, educational search engines based on free text searches can propose in the exploratory search interface the linguistic 
resources (thesaurus, ontology, etc.) on which based their indexing in the form of faceted, while adopting behavioral principles to display a custom facet according to the need of the student.

It may be that this comparison as well as findings and suggestions provided enough knowledge and will serve the researcher community a lot. It will involve them in working in this stage of research to develop educational search engines that respect the combinations of characteristics between exploratory search and educational search engine, thus evaluate them according to appropriate measures.

\section{CONCLUSION AND PERSPECTIVES}

The exploratory learning has an important place to ensure the acquisition of knowledge, understanding, interpretation of ideas, analyzes, synthesis and evaluation. It is ensured due to several technologies of online learning among them there are the educational search engines due exploratory search.

It is interesting to discuss the deep relations between the exploratory search and the educational search engines. This forms the sustainability of the exploratory search to educational search engines following their characteristics that rely on learning and investigative search goals.

Thus, the implementation of the exploratory search depends on educational search engine toward learning and the investigation of knowledge. The exploratory search is considered to disconnect with many students in search of knowledge if it does not focus on the learning and investigation of knowledge. This highlights the centrality of the search engine's relations with the student and the distinguishing belief of exploratory search in knowledge learning and investigating.

However, the question that arises; is the exploratory search always beneficial for the student; how the characteristics of exploratory search, for example personalized search, time, open-ended, can affect the satisfaction and the number of resources returned as well as the method followed in this exploratory operation. How to adopt exploratory search to search engines that does not support it for the moment. The question is open to the entire researcher community to find an answer that can improve exploratory search.

\section{BIODATA and CONTACT ADDRESSES of AUTHORS}

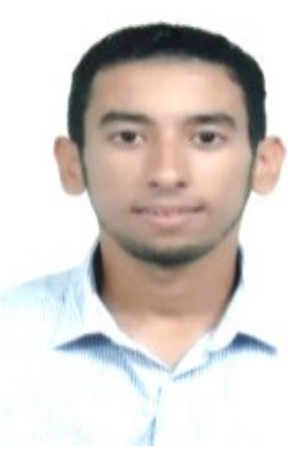

Dr. Kamal EL GUEMMAT got his PhD in computer sciences from the Faculty of Sciences Ben M'Sik, Hassan II University. He has several experiences in the field of engineering, teaching, training, and research. He has published some twenty articles in national, internationals conferences and journals. $\mathrm{He}$ does his research in Laboratory of Information Processing (LTI) in the Faculty of Sciences Ben M'Sik in which he develops his research in various fields. Also, he does research with other groups in different laboratories. His areas of research interest Semantic Indexing, Semantic Web, Information Retrieval Systems, Automatic Processing of Natural Language, Information and Communication Technology for Teaching and Learning, Educational Modeling, E-Learning.

\section{Dr. Kamal EL GUEMMAT}

Laboratory of Information Processing, Faculty of Sciences Ben M'Sik, Hassan II University

Address: Hassan II University, Faculty of Sciences Ben M'Sik, P.B. 7955, Casablanca, Morocco

Phone: +212614557142

E-mail: k.elguemmat@gmail.com 


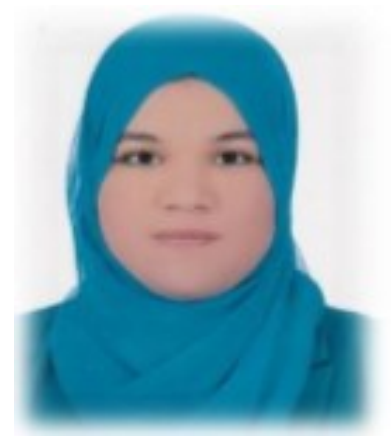

Dr. Sara OUAHABI was born in 1988 in Casablanca, Morocco. Took her Bachelor, Master, and $\mathrm{PhD}$ degree from Faculty of Sciences Ben M'Sik, Hassan II University. Her area of expertise is teaching, education and research. She develops her research in the Modeling and Information Processing Laboratory (LTIM) in the Faculty of Sciences Ben M'Sik with the collaboration of other research teams. She has participated in several national and international events and conferences and has published her work in international journals. Her interesting area includes: Cloud Computing, Distributed Learning, E-learning, Educational Modeling, Semantic Indexing, Information and Communication Technology for Teaching and Learning.

Dr. Sara OUAHABI

Modeling and Information Processing Laboratory, Faculty of Sciences Ben M'Sik, Hassan II University Address: Hassan II University, Faculty of Sciences Ben M'Sik, P.B. 7955, Casablanca, Morocco Phone: +212664140785

E-mail: sara.ouahabi@gmail.com

\section{REFERENCES}

Alhenshiri, A., Watters, C., Shepherd, M., \& Duffy, J. (2012). Building support for Web information gathering tasks. Proceedings of the 45th Hawaii International Conference on System Sciences (HICSS), 1687-1696.

Antoniou, G. van Harmelen. and F. (2004). A semantic web primer, The MIT Press chap. no. 1.

Aula, A., \& Russell, D.M. (2009). Complex and exploratory Web search. In Marchionini, G., et al. (organizers), Information Seeking Support Systems: An Invitational Workshop Sponsored by the National Science Foundation (June 26-27, 2008, Chapel Hill, NC), p23-24. Chapel Hill, NC: School of Information \& Library Science, University of North Carolina at Chapel Hill. http://ils.unc.edu/ISSS/ISSS_final_report.pdf.

Biletskiy, Y., Baghi, H., Steele, J., \& Vovk, R. (2012). A rule-based system for hybrid search and delivery of learning objects to learners. Interactive Technology and Smart Education, 9(4), 263-279.

Dabbagh, N. (2007). The online learner: Characteristics and pedagogical implications. Contemporary Issues in Technology and Teacher Education, 7(3), 217-226.

Dabbagh, N., \& Bannan-Ritland, B. (2005). Online learning: Concepts, strategies, and application. Upper Saddle River, NJ: Prentice Hall.

Department of Education, USA. (1993). Technologies for Exploratory Learning. [Online]. Available: http://www.ed.gov/pubs/EdReformStudies/TechReforms/chap2c.html [26th September 2001].

Diriye, A., Wilson, M.L., Blandford, A., \& Tombros, A. (2010). Revisiting exploratory search from the HCI perspective. HCIR 2010: Proceedings of the Fourth Workshop on Human-Computer Interaction and Information Retrieval. http://research.microsoft.com/en-us/um/people/ryenw/h cir2010/presentations.html.

Du, J.T., \& Evans, N. (2011). Academic users' information searching on research topics: Characteristics of research tasks and search strategies. Journal of Academic Librarianship, 37(4), 299-306.

El Guemmat, K., \& Ouahabi, S. (2018). A Literature Review of Indexing and Searching Techniques Implementation in Educational Search Engines. International Journal of Information and Communication Technology Education (IJICTE), 14(2), 72-83. 
El Guemmat, K., Benlahmar, E. H. Talea, M., \& Lamrani, E. K. (2015). Implementation and Evaluation of an Indexing Model of Teaching and Learning Resources. Procedia-Social and Behavioral Sciences, 191, 1266-1274.

Furini, M., Mirri, S., \& Montangero, M. (2017, July). TagLecture: The gamification of video lecture indexing through quality-based tags. In Computers and Communications (ISCC), 2017 IEEE Symposium on (pp. 122-127). IEEE.

Glowacka, D., Ruotsalo, T., Konuyshkova, K., Kaski, S., \& Jacucci, G. (2013, March). Directing exploratory search: Reinforcement learning from user interactions with keywords.

In Proceedings of the 2013 international conference on Intelligent user interfaces (pp. 117-128). ACM.

Gluz, J. C., Silveira, E. L., da Silva, L. R. J., \& Barbosa, J. L. V. (2016). Towards a Semantic Repository for Learning Objects: Design and Evaluation of Core Services. Journal of Universal Computer Science, 22(1), 16-36.

Hassan, S. AND Mihalcea, R. (2009). Learning to identify educational materials. In Proceedings of the Conference on Recent Advances in Natural Language Processing (RANLP).

Ip, A., \& Naidu, S. (2001). Reuse of Web-Based Resources in Technology-Enhanced StudentCentered Learning Environments. Campus Wide Information Systems.

Ip, A., Morrison, I., Currie, M., \& Mason, J. (2000). Managing Online Resources for Teaching and Learning. Paper presented at the AusWeb2K, the Six Australian World Wide Web Conference.

Jansen, B.J., Booth, D., \& Smith, B. (2009). Using the taxonomy of cognitive learning to model online searching. Information Processing \& Management, 45(6), 643-663.

Kammerer, Y., Nairn, R., Pirolli, P., \& Chi, E. H. (2009, April). Signpost from the masses: learning effects in an exploratory social tag search browser. In Proceedings of the SIGCHI conference on human factors in computing systems (pp. 625-634). ACM.

Kim, J., 2009 Describing and predicting information-seeking behavior on the Web. Journal of the American Society for Information Science \& Technology, 60(4): 679-693.

Kintsch, W. (1998) Comprehension: A Paradigm for Cognition. New York: Cambridge University Press.

Klouche, K., Ruotsalo, T., Cabral, D., Andolina, S., Bellucci, A., \& Jacucci, G. (2015, April). Designing for exploratory search on touch devices. In Proceedings of the 33rd Annual ACM Conference on Human Factors in Computing Systems (pp. 4189-4198). ACM.

Lee, M. C., Tsai, K. H., \& Wang, T. I. (2008). A practical ontology query expansion algorithm for semantic-aware learning objects retrieval. Computers \& Education, 50(4), 1240-1257.

Li, Y. (2009). Exploring the relationships between work task and search task in information search. Journal of the American Society for Information Science \& Technology, 60(2), 275-291.

Marchionini, G. 2006. Exploratory Search: From Finding to Understanding. CACM 49(4): 41-46.

Marchionini, G., Dwiggins, S., Katz, A., \& Lin, X. (1993) Information seeking in full-text end-useroriented search systems: The roles of domain and search expertise. Library \& Information Science Research, 15(1), 35-69.

Miwa, M., \& Kando, N. (2007). Methodology for capturing exploratory search processes. In Proceeding of the ACM SIGCHI 2007 Workshop on Exploratory Search and HCI: Designing and Evaluating Interfaces to Support Exploratory Search Interaction (ESI2007) (pp. 76-80). 
Halvey, M., Vallet, D., Hannah, D., M. Jose, J. (2014) Supporting exploratory video retrieval tasks with grouping and recommendation, Information Processing \& Management, Volume 50, Issue 6, 2014, Pages 876-898.

Niu, Y., \& Winter, S. (2006). The effect of task type and information format on Web searching performance. ICIS Proceedings. Paper 52. http://aisel.aisnet.org/icis2006/52.

PIROLLI, P., AND CARD, S. Information foraging in information access environments. In Proc. ACM SIGCHI (New York, NY, USA, 1995), ACM Press/Addison-Wesley Publishing Co., pp. 51-58.

Qu, Y., \& Furnas, G.W. (2008). Model-driven formative evaluation of exploratory search: A study under a sensemaking framework. Information Processing \& Management 44(2): 534-555.

Qu, Y., \& Furnas, G.W. (2006). What do the attributes of exploratory search tell us about evaluation. Proceedings of the ACM SIGIR 2006 Workshop on Evaluating Exploratory Search Systems (EESS), 51-53.

Rahman, M. M., Abdullah, N. A., \& Aurangozeb, F. (2017, May). A framework for designing a personalised web-based search assistant tool for eLearning. In Information and Communication Technology (ICoIC7), 2017 5th International Conference on (pp. 1-6). IEEE.

Ramdeen, S., \& Hemminger, B. (2012). A tale of two interfaces: How facets affect the library catalog search experience. Journal of the American Society for Information Science \& Technology, 63(4), 702-715.

Shneiderman, B. and Plaisant, C. Designing the User Interface 4th Ed. Person/Addison-Wesley, Boston, MA, 2005.

Smine, B., Faiz, R., \& Desclés, J. P. (2011). A semantic annotation model for indexing and retrieving learning objects. JDIM, 9(4), 159-166.

Toms, E.G., O'Brien, H., Mackenzie, T., Jordan, C., Freund, L., Toze, S., Dawe, E., \& MacNutt, A. (2008). Task effects on interactive search: The query factor. In Focused Access to XML Documents. Lecture Notes in Computer Science, 4862, 359-372.

Vakkari, P. (2010). Exploratory searching as conceptual exploration. HCIR 2010: Proceedings of the Fourth Workshop on Human-Computer Interaction and Information Retrieval.

Valiente, M. C., Sicilia, M. A., Garcia-Barriocanal, E., \& Rajabi, E. (2015). Adopting the metadata approach to improve the search and analysis of educational resources for online learning. Computers in Human Behavior.

White, R., \& Roth, R.A. (2009). Exploratory Search: Beyond the Query-Response Paradigm. Synthesis Lectures on Information Concepts, Retrieval, and Services \#3. Morgan \& Claypool.

WHITE, R. W., KULES, B., DRUCKER, S. M., AND M.C. SCHRAEFEL. Introduction. Commun. ACM 49, 4 (2006a), 36-39.

White, R., Muresan, G., \& Marchionini, G. (2006b). Proceedings of the ACM SIGIR 2006 Workshop on "Evaluating Exploratory Search Systems."

White, M.D., \& Iivonen, M. (2001). Questions as a factor in Web search strategy. Information Processing \& Management, 37(5), 721-740.

Wildemuth, B. M., \& Freund, L. (2012, October). Assigning search tasks designed to elicit exploratory search behaviors. In Proceedings of the Symposium on Human-Computer Interaction and Information Retrieval (p. 4). ACM. 
Wildemuth, B. M. (2007). Subjectivity: Its Role in Exploratory Search Processes and Evaluation. ESI 2007, 96.

Wildemuth, B.M., de Blied, R., Friedman, C.P., and File, D.D. Medical students' personal knowledge, searching proficiency, and database use in problem solving. Journal of the American Society for Information Science, 46 (1995), 590-607.

Wilson, M. L. (2007). Bridging the gap: Using IR models for evaluating exploratory search interfaces.

Wolfram, D., \& Dimitroff, A. (1998). Hypertext vs. Boolean-based searching in a bibliographic database environment: A direct comparison of searcher performance. Information Processing \& Management, 34(6), 669-680.

Wu, W.-C., Kelly, D., Edwards, A., \& Arguello, J. (In press). Grannies, tanning beds, tattoos and NASCAR: Evaluation of search tasks with varying levels of cognitive complexity. Paper accepted for presentation at Information Interaction in Context (IIiX) 2012.

Zhao, J., Kan, M. Y., \& Theng, Y. L. (2008). Math information retrieval: user requirements and prototype implementation. In Proceedings of the 8th ACM/IEEE-CS joint conference on Digital libraries (pp. 187-196). 